\title{
Analysis on Human Blockage Path Loss and Shadow Fading in Millimeter-Wave Band
}

\author{
Hongmei Zhao, Qian Wang, and Kunfeng Shi \\ College of Electric and Information Engineering, Zhengzhou University of Light Industry, Zhengzhou 450002, China \\ Correspondence should be addressed to Qian Wang; 1002818773@qq.com
}

Received 29 May 2017; Accepted 12 July 2017; Published 20 August 2017

Academic Editor: Francisco Falcone

Copyright (C) 2017 Hongmei Zhao et al. This is an open access article distributed under the Creative Commons Attribution License, which permits unrestricted use, distribution, and reproduction in any medium, provided the original work is properly cited.

Millimeter-wave (Mm-w) is the trend of communication development in the future; users who carry mobile communication equipment could be blocked by others in a crowded population environment. Based on Shooting and Bouncing Ray (SBR) method and setting up different orientation receivers (RX), population density, and people fabric property at $28 \mathrm{GHz}$ and $38 \mathrm{GHz}$, simulating experimental scene similar to station square by Wireless Insite software, we use least square method to do linear-regression analysis for path loss and build path loss model. The result shows that the path loss index has a certain change in the different frequency, orientation receivers, population density, and people fabric. The path loss index of RouteC1 and RouteA2 has an obvious change in the central transmitter (TX). Each route shadow fading obeys Gaussian distribution whose mean is 0 . This paper's result has a theoretical guiding for designing the communication system in a crowded population environment.

\section{Introduction}

With the increase of smart phone users and the development of mobile APP, requirement for wireless data transmission rate is on daily increase. The frequency spectrum resource is very crowded in original; it cannot satisfy these requirements and is badly in need of new frequency spectrum to satisfy this one requirement. Now, Mm-w $(30 \mathrm{GHz}-300 \mathrm{GHz})$ starts to catch people's eyes; more and more industry and application come to use Mm-w frequency. For some population quite dense areas like station, playground, and so on, human body is the one main blockage of influence signal transmission, and the different fabric could have some influences. When $\mathrm{RX}$ position and direction are different, path loss also has different change.

The effect of population density is the one key of radio wave propagation characteristics outdoor. The authors in [1] have done some researches for throughput capacity of different population density $(\rho)$ in $60 \mathrm{GHz}$ outdoor campus, $\rho$ is from 0 to 0.01 , and the throughput capacity change is less. When $\rho$ is bigger, it means that, in particularly crowded environment, throughput capacity is toboggan. But it does not consider the electromagnetic property of people clothes. The work in $[2,3]$ had actual measurement for urban high-rise and low-rise in $28 \mathrm{GHz}$ outdoor, analyzes the actual measurement data, and gets the path loss index and shadow fading by using the path loss model relevant to distance. However, considering $\mathrm{Mm}-\mathrm{w}$ research it is more in indoor environment; the research on human blockage outdoor is fewer and fewer. The work in [4] had research on wireless communication channel parameters and the dependency in $60 \mathrm{GHz}$ indoor; analysis of channel parameters includes multipath number, RMS delay spread, rice factor, shadow fading characteristics, and distribution model.

This paper uses SBR as theoretical basis, to simulate the likeness station square by Wireless Insite and get the path loss. Modeling path loss by least square method is to obtain path loss model and shadow fading in different route.

\section{Simulation Environment}

2.1. Scene Model. We build simulation scene plans similar to station square, as shown in Figure 1(a). It distributes 3-6-floor buildings at two sides of square, and buildings are uniform rectangular. Human model is shown in Figure 1(b); it has two parts, head and body; all 8 sides are cubes. Head height and radius, respectively, are $0.2 \mathrm{~m}$ and $0.08 \mathrm{~m}$, body radius is $0.25 \mathrm{~m}$, and height is uniform distributed between $1.3 \mathrm{~m}$ and 


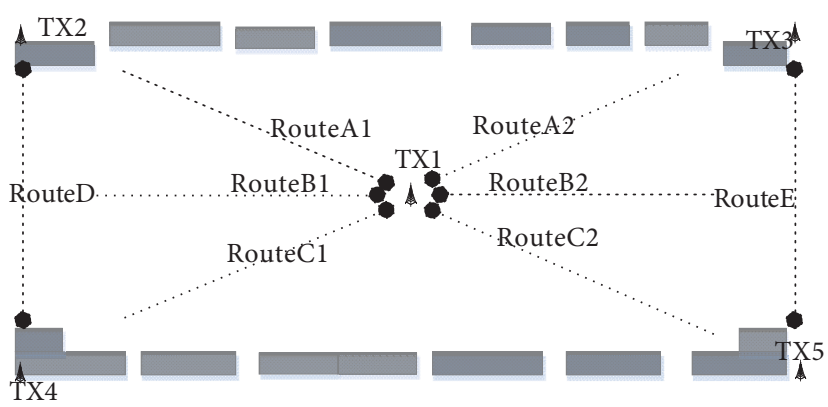

(a) Simulation scene

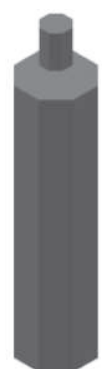

(b) Human model

FIgURE 1: Simulation scene and human model.

TABLE 1: TX/RX parameters.

\begin{tabular}{lcc}
\hline & TX & RX \\
\hline High $(\mathrm{m})$ & 30 & 1.5 \\
Gain $(\mathrm{dBi})$ & 21.6 & 0 \\
$\begin{array}{l}\text { Transmitted power } \\
(\mathrm{dBm})\end{array}$ & 10 & - \\
Type & $12 * 12$ array & $4 * 4$ array \\
Polarization & Vertical polarization & Vertical polarization \\
\hline
\end{tabular}

$1.6 \mathrm{~m}$. Head and body electromagnetic parameter set up skin and different clothes (cotton, red leather, and yellow leather) respectively, meaning that we consider the effect of human fabric. Human is set at the station square randomly, the area is $500 * 170 \mathrm{~m}^{2}$, and simulation scene study area is $500 * 230 \mathrm{~m}^{2}$. When $\rho$ is $0.01 / \mathrm{m}^{2}$, the number of people is 1150 in simulation scene; when $\rho$ is $0.1 / \mathrm{m}^{2}$, the number of people is 11500 .

2.2. Design on $T X / R X$. There are $5 \mathrm{TX}$ in simulation scene; four corners have each one and center has one. Center TX1 has 6 receive routes, that is, RouteA1, RouteB1, RouteC1, RouteA2, RouteB2, and RouteC2; each route length is $180 \mathrm{~m}$, starting from TX1 to $20 \mathrm{~m}$; RouteA1, RouteC1, RouteA2, and RouteC 2 horizontal angle are $30^{\circ}$. TX2, TX4 and TX3, TX5 receive route, respectively, are RouteD and RouteE in vertical direction; the length is $170 \mathrm{~m}$; the other receive route is RouteA1, RouteC1, RouteA2, and RouteC2, respectively, in four corners. RX distance is $3 \mathrm{~m}$ in 8 receive routes; TX and $\mathrm{RX}$ antenna parameters are set as shown in Table 1.

2.3. Other Parameters' Setting. The simulated wave is set up sinusoid; the bandwidth is $1 \mathrm{GHz}$ in $28 \mathrm{GHz}$ and $38 \mathrm{GHz}$; each material property is shown in Table $2[5,6]$. The wall thickness is $0.38 \mathrm{~m}$, roughness is $0.001 \mathrm{~m}$, and human skin and clothes roughness are $0 \mathrm{~m}$.

Similarity to the station square simulation scene is shown in Figure 2.
TABLE 2: Materials property parameters.

\begin{tabular}{lcccc}
\hline \multirow{2}{*}{ Materials } & \multicolumn{2}{c}{$\varepsilon$} & \multicolumn{2}{c}{$\sigma$} \\
& $28 \mathrm{GHz}$ & $38 \mathrm{GHz}$ & $28 \mathrm{GHz}$ & $38 \mathrm{GHz}$ \\
\hline Skin & 10.7 & 10.05 & 7.32 & 10.25 \\
Cotton & 1.7 & 1.56 & 0.038 & 0.12 \\
Red leather & 2.15 & 2.04 & 0.14 & 0.23 \\
Yellow leather & 2.3 & 2.12 & 0.09 & 0.067 \\
Concrete & 6.23 & 6.20 & 0.2 & 0.22 \\
\hline
\end{tabular}

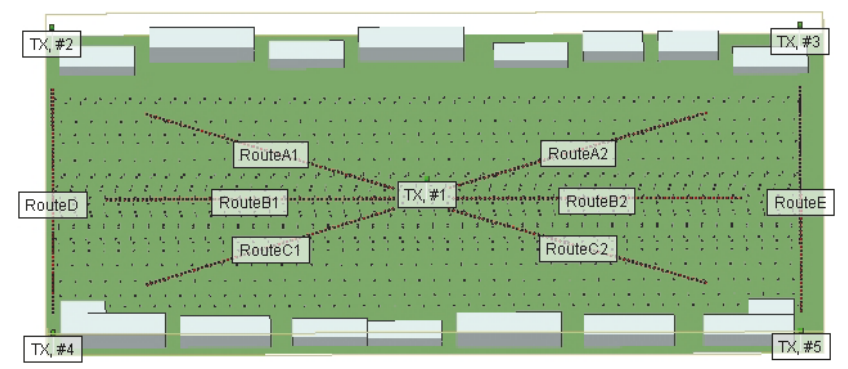

Figure 2: Similarity to the station square simulation scene.

\section{Path Loss}

Simplified path loss model formula is as follows:

$$
P_{R}[\mathrm{dBm}]=P_{T}[\mathrm{dBm}]+K[\mathrm{~dB}]-10 \cdot n \cdot \lg \left(\frac{d}{d_{0}}\right) .
$$

$P_{R}$ is receive power, $P_{T}$ is transmit power, $n$ is path loss index, $d$ is distance between TX and RX, $d_{0}$ is reference, in general, $d_{0}=1 \mathrm{~m}$, and $K$ usually is the free space path gain in $d_{0}$, where

$$
K=\mathrm{PG}\left(d_{0}\right)=-\mathrm{PL}\left(d_{0}\right)
$$

Bring (2) into (1):

$$
P_{R}=P_{T}-\mathrm{PL}\left(d_{0}\right)-10 \cdot n \cdot \lg \left(\frac{d}{d_{0}}\right) .
$$


Define channel path loss as path loss truth-value decibel, where $P_{R}$ and $P_{T}$ are $\mathrm{dB}$ difference; the formula is

$$
\mathrm{PL}(d)=10 \lg \left(\frac{P_{T}}{P_{R}}\right) .
$$

Bring (3) into (4), and, to take logarithm on both sides,

$$
\mathrm{PL}(d)=\mathrm{PL}\left(d_{0}\right)+10 \cdot n \cdot \lg \left(\frac{d}{d_{0}}\right) .
$$

Because actual wireless communication environment is more complex, receive signal is superposition by reflect, diffraction, transmission, and the other taken attenuation characteristics multipath signal; it is different from freedom space. In multipath, it always uses mixed model between path loss and shadow fading; the formula is

$$
\mathrm{PL}(d)=\mathrm{PL}\left(d_{0}\right)+10 \cdot n \cdot \lg \left(\frac{d}{d_{0}}\right)+X_{\sigma} .
$$

$X_{\sigma}$ is Gaussian random variable whose mean is 0 and variance is $\sigma^{2}$.

\subsection{Human Blockage Path Loss in 28 GHz Different Population} Density. According to receive route directivity, the receive routes RouteA1, RouteB1, RouteC1, RouteA2, RouteB2, and RouteC2 are selected in TX1, TX2, TX4, and TX3; TX5 receive route is RouteD and RouteE in vertical direction, respectively; TX2, TX3, TX4, and TX5 receive routes are RouteA1, RouteC1, RouteA2, and RouteC2, respectively.

That could obtain the path loss data of receive route RouteA1 in TX1 when population density is $0.01 / \mathrm{m}^{2}$ (human fabric is cotton) in $28 \mathrm{GHz}$ by Wireless Insite, for getting $\operatorname{PL}\left(d_{0}\right)$ and path loss index $n$; let $\operatorname{PL}(d)=Y$, $\operatorname{PL}\left(d_{0}\right)=a, 10 \lg \left(d / d_{0}\right)=x$. For $N$ pairs of observations $\left(x_{1}, y_{1}\right),\left(x_{2}, y_{2}\right), \ldots,\left(x_{N}, y_{N}\right)$, formula (6) changes to

$$
Y_{i}=a+n x_{i}+X_{\sigma i}
$$

$X_{\sigma i}$ expresses the $i$ times observation random deviation. Regression analysis was performed on data by the least square method, $a=65.57, n=3.02$ (Figure 3, scatter-fitting line). When $\rho$ is $0.01 / \mathrm{m}^{2}$ in $28 \mathrm{GHz}$, receive route RouteAl path loss model formula in TX1 is

$$
\mathrm{PL}(d)=65.57+10 \cdot 3.02 \cdot \lg \left(\frac{d}{d_{0}}\right)+X_{\sigma}
$$

$(d<200 \mathrm{~m})$.

In $\mathrm{TX} 1$, regression analysis on 6 receive routes in different population density obtains path loss index as shown in Figure 4.

With the increase of population density, RouteA1, RouteB1, RouteC1, and RouteB2 path loss index all are decreased; RouteA2 and RouteC 2 are increased; RouteC1 and RouteA2 path loss index $n$ are less than freedom space path loss index $(n=2)$. When TX is located in RX northeast $30^{\circ}$ and southwest $30^{\circ}, n<2$, compared with other azimuth $\mathrm{RX}, n$ is mix, path loss $\operatorname{PL}\left(d_{0}\right)$ which as $d_{0}=1 \mathrm{~m}$ is reference distance

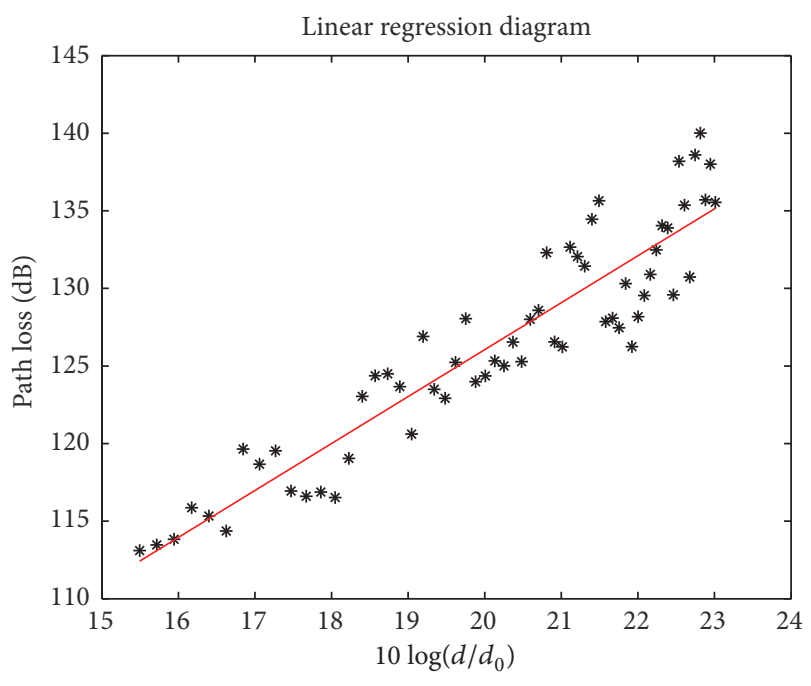

FIGURE 3: Scatter-fitting line in $28 \mathrm{GHz}$.

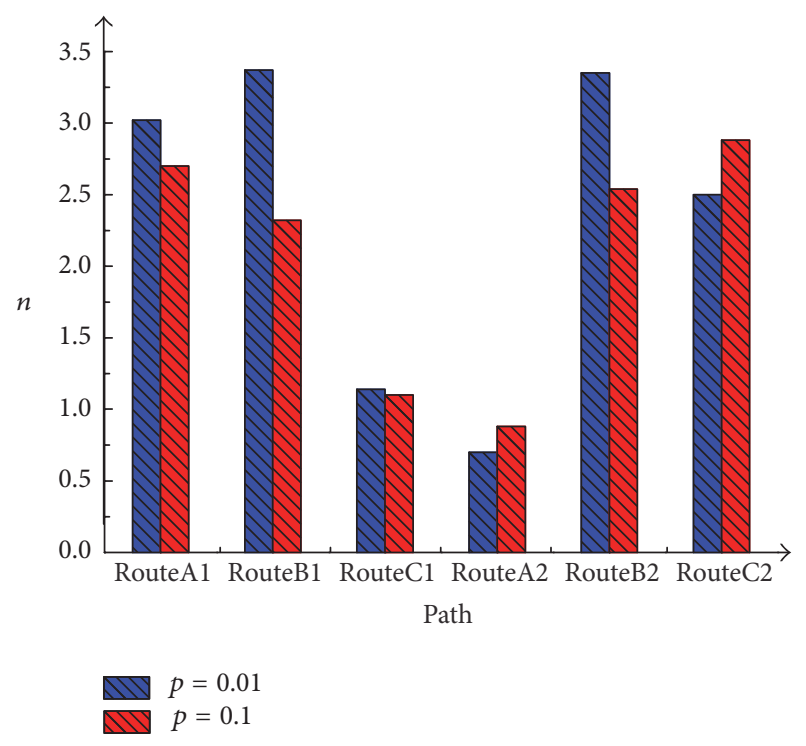

FIgUre 4: Each path loss index in $28 \mathrm{GHz}$ TX1.

and is the largest. When $\rho$ is $0.01 / \mathrm{m}^{2}$, path loss max differs is $60 \mathrm{~dB}$; mix also is $30 \mathrm{~dB}$, when $\rho$ is $0.1 / \mathrm{m}^{2}$; path loss differs around $30 \mathrm{~dB}$.

When $\rho$ is $0.01 / \mathrm{m}^{2}$, RouteC1 scatter-fitting line in TX4 as shown in Figure 5, gets $a=169.58, n=-1.26$.

When $\rho=0.01 / \mathrm{m}^{2}$ at $28 \mathrm{GHz}$, RouteC1 path loss model formula in TX4 is

$$
\mathrm{PL}(d)=169.58+10 \cdot(-1.26) \cdot \lg \left(\frac{d}{d_{0}}\right)+X_{\sigma}
$$

$$
(d<200 \mathrm{~m}) .
$$

Each of TX2, TX3, TX4, and TX5 receives route path loss regression analysis as shown in Table 3. 
TABLE 3: TX2, TX3, TX4, and TX5 each receive route $a$ and $n$.

\begin{tabular}{lccccccccccccccccc}
\hline TX & \multicolumn{4}{c}{ TX2 } & \multicolumn{4}{c}{ TX3 } & \multicolumn{4}{c}{ TX4 } & \multicolumn{4}{c}{ TX5 } \\
Route & \multicolumn{2}{c}{ RouteA1 } & \multicolumn{2}{c}{ RouteD } & \multicolumn{2}{c}{ RouteA2 } & \multicolumn{2}{c}{ RouteE } & \multicolumn{2}{c}{ RouteC1 } & RouteD & RouteC2 & RouteE \\
\hline$\rho$ & 0.01 & 0.1 & 0.01 & 0.1 & 0.01 & 0.1 & 0.01 & 0.1 & 0.01 & 0.1 & 0.01 & 0.1 & 0.01 & 0.1 & 0.01 & 0.1 \\
$a$ & 114.04 & 88.8 & 40.13 & 37.36 & 72.09 & 80.92 & 48.62 & 74.53 & 169.58 & 188.57 & 38.3 & 37.94 & 211.47 & 271.67 & 98.13 & 54.28 \\
$n$ & 1.24 & 2.16 & 3.75 & 3.88 & 3.18 & 2.52 & 3.35 & 2.17 & -1.26 & -2.36 & 3.83 & 3.85 & -3.2 & -6.36 & 3.77 & 3.06 \\
\hline
\end{tabular}

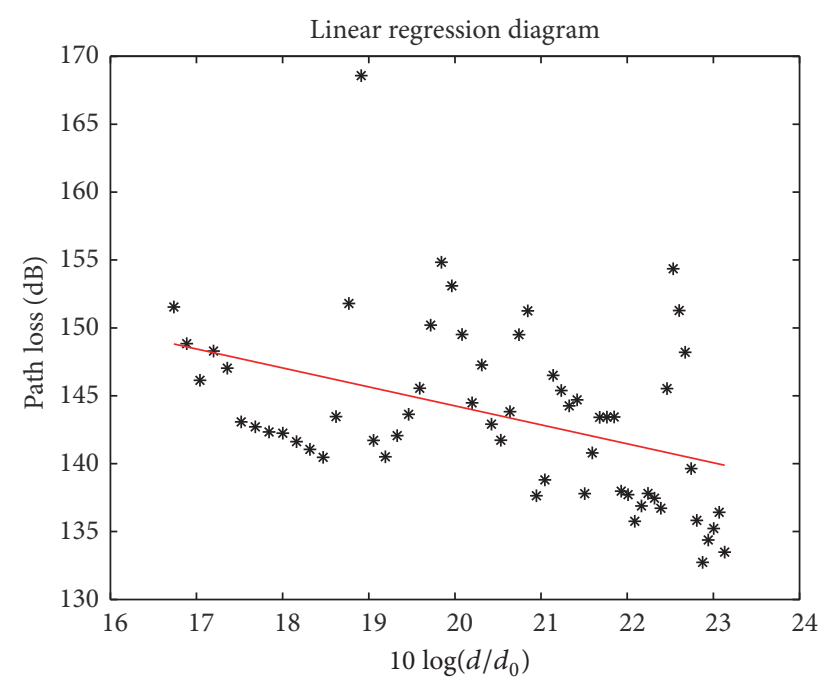

FIGURE 5: RouteCl scatter-fitting line in $0.01 / \mathrm{m}^{2} \mathrm{TX} 4$.

From Table 3, we know that RouteC1 and RouteC2 path loss index all are negative in TX4 and TX5, and as population density gets bigger, the path loss index will be thinner in these two routes. With increase of population density, the path loss index in TX2 is increases, only in $\rho=0.01 / \mathrm{m}^{2}, n<2$ in RouteA1. RouteD path loss index amplification is less in TX4, only 0.2. Different azimuth RX has different path loss index, and $\rho$ differs; the effect is also different by different azimuth $\mathrm{RX}$.

3.2. Human Blockage Path Loss in 38 GHz Different Population Density. The simulation is similar to the method which, in $28 \mathrm{GHz}$, change in the frequency is $38 \mathrm{GHz}$; bandwidth still is $1 \mathrm{GHz}$; considering the human fabric is only cotton, simulating $\rho$ is $0.01 / \mathrm{m}^{2}$ and $0.1 / \mathrm{m}^{2}$ in $38 \mathrm{GHz}$. As shown in Figure 6, when $\rho$ is different, each receives route path loss index tendency chart in TX1.

Compared with $28 \mathrm{GHz}$, the path loss index $n$ trend is opposite with population density changing in RouteAl and RouteCl, with $\rho$ increasing and $n$ decreasing. $n$ is less than 2 in RouteA1, RouteC1, RouteA2, and RouteC 2 and $n$ is greater than 2 in RouteCl, $\rho=0.1 / \mathrm{m}^{2}$.

When $\rho$ is $0.01 / \mathrm{m}^{2}, 38 \mathrm{GHz}$ compared with $28 \mathrm{GHz}, n$ is bigger in RouteA2 and RouteCl; the other is less. When $\rho$ is $0.1 / \mathrm{m}^{2}$, except RouteA2 and RouteCl, it is also bigger in RouteB2 and RouteC2.

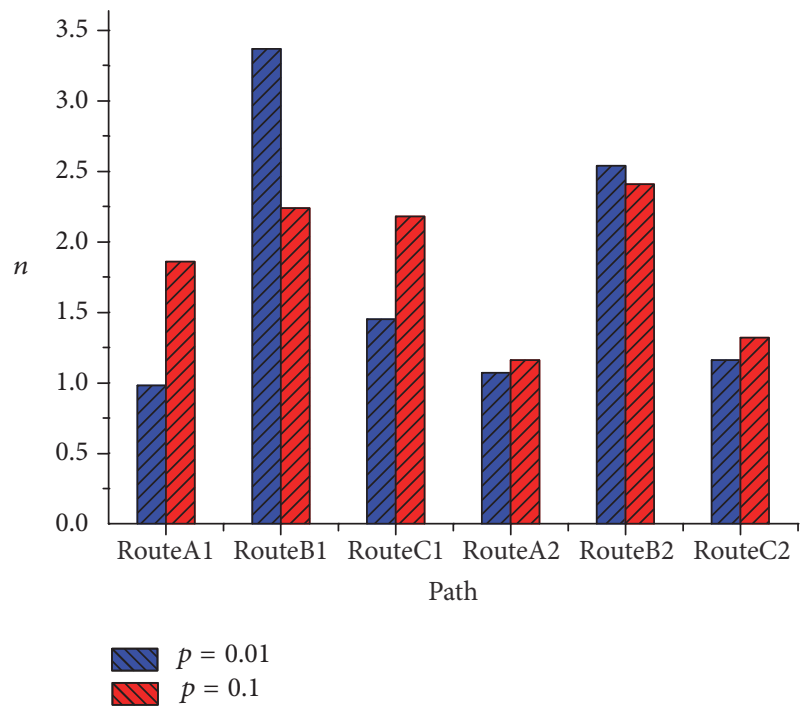

FIGURE 6: Each path loss index in $38 \mathrm{GHz}$ TX1.

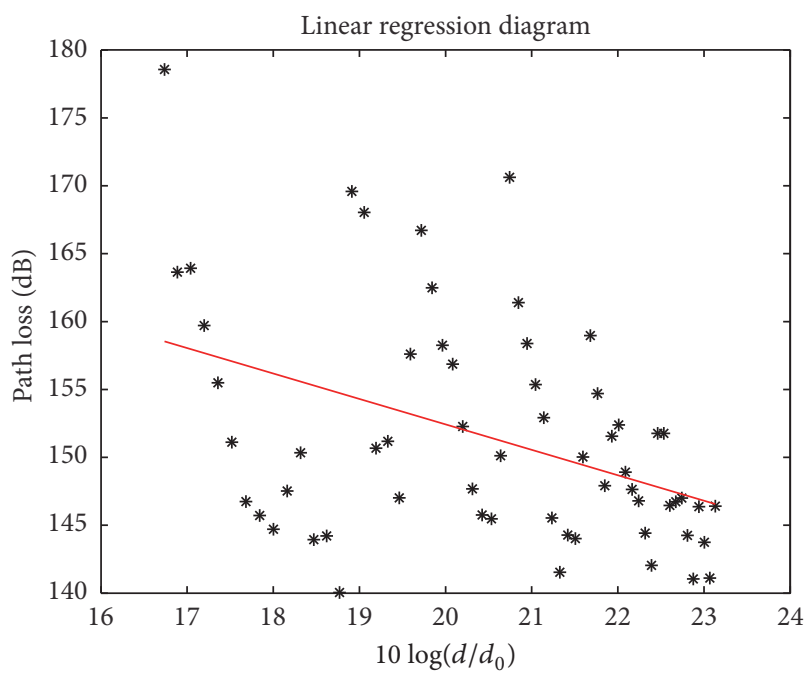

Figure 7: RouteC1 scatter-fitting line in $0.1 / \mathrm{m}^{2}$ TX4.

TX2, TX3, TX4, and TX5, located in four corners, are similar path loss index changing trend to $28 \mathrm{GHz}$; the only difference is that RouteAl is increased with population density in TX2 and $n$ is increased, when $\rho$ is $0.01 / \mathrm{m}^{2}, n>$ 2. Identically, RouteCl and RouteC2 path loss index $n$ are negative in TX4 and TX5. As shown in Figure 7, when $\rho$ is 
TABLE 4: Each receive route $a$ and $n$ in $28 \mathrm{GHz}$ and $38 \mathrm{GHz}$.

\begin{tabular}{lcccc}
\hline & \multicolumn{2}{c}{$28 \mathrm{GHz}$} & \multicolumn{2}{c}{$38 \mathrm{GHz}$} \\
& $a$ & $n$ & $a$ & $n$ \\
\hline RouteA1 & 78.96 & 2.46 & 94.70 & 1.76 \\
RouteB1 & 72.36 & 2.11 & 80.50 & 1.88 \\
RouteC1 & 93.98 & 1.43 & 81.97 & 2.41 \\
RouteA2 & 109.73 & 0.63 & 107.59 & 1.21 \\
RouteB2 & 70.67 & 2.12 & 72.13 & 2.31 \\
RouteC2 & 62.01 & 3.14 & 104.71 & 1.29 \\
\hline
\end{tabular}

$0.1 / \mathrm{m}^{2}$, RouteC1 scatter-fitting line in TX4 gets $a=188.42$, $n=-1.80$.

Path loss model formula in TX4 where $\rho$ is $0.1 / \mathrm{m}^{2}$ in $38 \mathrm{GHz}$ is

$$
\mathrm{PL}(d)=188.42+10 \cdot(-1.80) \cdot \lg \left(\frac{d}{d_{0}}\right)+X_{\sigma}
$$

$$
(d<200 \mathrm{~m}) \text {. }
$$

\subsection{Different Fabric Human Blockage Path Loss in $28 \mathrm{GHz}$ and} $38 \mathrm{GHz}$. Human fabric varies, leading to radio wave getting some change. In building simulation scene, the human fabric electromagnetic parameters set up cotton, red leather, and yellow leather, and they are distributed uniformly. When $\rho$ is $0.1 / \mathrm{m}^{2}$, each path loss index from regression analysis to $a$ and $n$ in TX1 is shown in Table 4 .

Compared with human wearing cotton fabric, in different receive orientations, path loss index has different change. At $28 \mathrm{GHz}, n$ is less than the single cotton that only in RouteC1 and RouteC2 these two receive routes and other routes $n$ are all bigger. At $38 \mathrm{GHz}$, RouteC1 and RouteA 2 are less than the single cotton.

\section{Shadow Fading}

After linear-regression analysis on each route, we also need verification for shadow fading distribution characteristics. Considering human wear fabric variety, when $\rho$ is $0.1 / \mathrm{m}^{2}$ in $28 \mathrm{GHz}$, such as RouteC1 data in TX1, bring $a$ and $n$ into (7), which could get $x_{\sigma 1}, x_{\sigma 2}, \ldots, x_{\sigma N}$. Use for $\chi^{2}$ goodnessof-fit test method, shadow fading obeys normal distribution whose mean is $\mu$ and variance is $\sigma^{2}$ in general, so we have the assumption $H_{0}: X_{\sigma} \sim N\left(\mu, \sigma^{2}\right)$. Because parameter is unknown, usually, that needs maximum likelihood method to estimate $\mu$ and $\sigma$ [7].

$$
\begin{aligned}
& \mu=\frac{1}{N} \sum_{i=1}^{N} x_{\sigma i}=0, \\
& \sigma=\sqrt{\frac{1}{N} \sum_{i=1}^{N}\left(x_{\sigma i}-\tilde{x}\right)^{2}}=4.84 .
\end{aligned}
$$

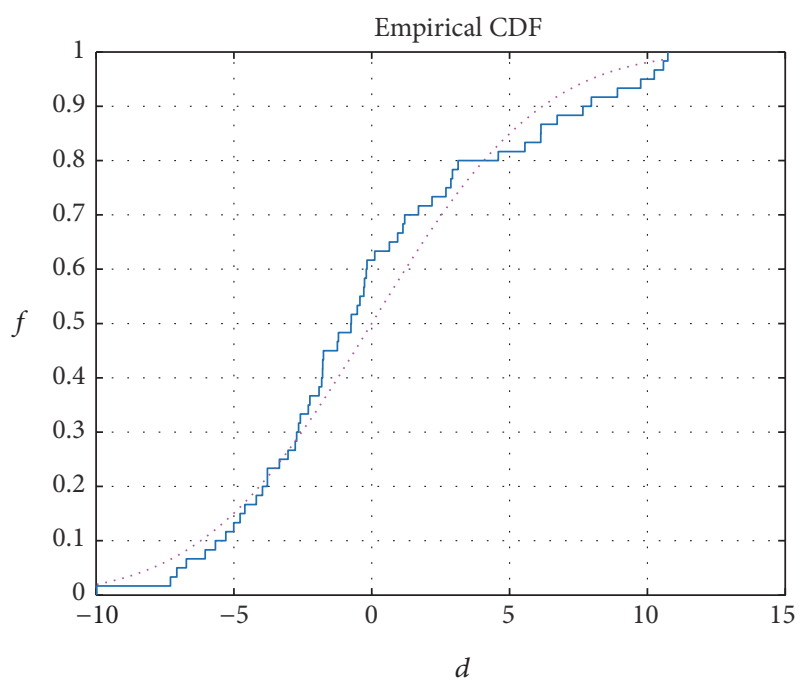

..... Theories normal distribution - Empirical distribution

FIGURE 8: Empirical distribution and theories normal distribution.

Establish that totality is only extracting $m$ discrete type random variable, without loss of generality; $X$ probable value is $1,2, \ldots, m$. Record $X$ value $i$ 's probability $p_{i}$; that is,

$$
P(X=i)=p_{i} \quad i=1,2,3, \ldots, m
$$

obviously,

$$
\sum_{i=1}^{m} p_{i}=1
$$

According to law of great numbers, frequency is reflection of probability; global probability distributions are $\left(p_{0}, p_{1}, \ldots, p_{m 0}\right)$,

$$
\chi^{2}=\sum_{i=1}^{m} \frac{\left(n_{i}-n p_{i 0}\right)^{2}}{n p_{i 0}} \approx \chi^{2}(m-1) .
$$

When observed value $\chi_{0}{ }^{2}=\sum_{i=1}^{m}\left(\left(n_{i}-n p_{i 0}\right)^{2} / n p_{i 0}\right)$ is less than $\chi_{\alpha}^{2}(m-1)$ of given significance level $0<\alpha<1$, it accepts assumption $H_{0}$. For $\alpha=0.05$, degree for freedom is 5; looking up $\chi_{0.05}^{2}(5)=11.07, \chi_{0}{ }^{2}=9.02$, so $\chi_{0}^{2}<\chi_{\alpha}^{2}(m-1)$; accept $H_{0}$; that is, $X_{\sigma} \sim N\left(0,4.84^{2}\right)$; empirical distribution and theories of normal distribution are shown in Figure 8.

For these routes' shadow fading, use $\chi^{2}$ to test and find that they all obey normal distribution whose mean is 0 , and considering human fabric variety, each TX1 route's shadow 
fading distribution that $\rho$ is $0.1 / \mathrm{m}^{2}$ in $28 \mathrm{GHz}$ and $38 \mathrm{GHz}$, respectively, is

$$
28 \mathrm{GHz}: X_{\sigma} \sim \begin{cases}N\left(0,6.26^{2}\right), & \text { RouteA1 } \\ N\left(0,5.33^{2}\right), & \text { RouteB1 } \\ N\left(0,4.84^{2}\right), & \text { RouteC1 } \\ N\left(0,4.22^{2}\right), & \text { RouteA2 } \\ N\left(0,5.24^{2}\right), & \text { RouteB2 } \\ N\left(0,2.56^{2}\right), & \text { RouteC2 }\end{cases}
$$

\section{Conclusion}

Millimeter-wave is the trend for communication development in the future; in the crowded people environment, mobile communication equipment taken by users could be blocked by others. Based on SBR, to simulate experiment scene by Wireless Insite, it can get path loss and regression analysis through least square method and build path loss model. The result shows that the 6 receive routes of TX1, in the common conditions, RouteC1 and RouteA2, are opposite to others; when $\rho$ is certain, these two routes $n$ are decreased while frequency is increased. When $f$ and $\rho$ are certain, human fabric sets up diversify; RouteC1 and RouteA2 are also decreased. $n$ is $1.10 \sim 2.41$ in RouteCl; it is $0.63 \sim 1.16$ in RouteA2, and all is less than freedom space path loss index $(n=2)$. When frequency, population density, and human wear fabric diversity are certain, receive routes $\mathrm{C} 1$ and $\mathrm{C} 2$ path loss index all are negative in TX4 and TX5. Each shadow fading obeys normal distribution whose mean is 0 . This result has some theoretical direction significance for communication system design in crowded population environment.

\section{Conflicts of Interest}

The authors declare that there are no conflicts of interest regarding the publication of this paper.

\section{Acknowledgments}

This paper is supported by the Joint Funds of National Natural Science Foundation of China (U1504604) and supported by the Graduate's Scientific Research Foundation of Zhengzhou University of Light Industry (2016045).

\section{References}

[1] M. Abouelseoud and G. Charlton, "The effect of human blockage on the performance of millimeter-wave access link for outdoor coverage," in Proceedings of the 2013 IEEE 77th Vehicular Technology Conference, VTC Spring 2013, IEEE, Dresden, Germany, 2-5 June 2013.

[2] M. D. Kim, J. Liang, Y. K. Yoon, and J. H. Kim, “28GHz Path Loss Measurements in Urban Environments using Wideband Channel Sounder,' in Proceedings of the 2015 IEEE International Symposium on Antennas and Propagation \& USNC/URSI National Radio Science Meeting, IEEE, Vancouver, BC, Canada, 19-24 July 2015.

[3] M.-D. Kim, J. Liang, J. Lee, J. Park, and B. Park, "Directional multipath propagation characteristics based on $28 \mathrm{GHz}$ outdoor channel measurements," in Proceedings of the 10th European Conference on Antennas and Propagation, EuCAP 2016, IEEE, Davos, Switzerland, 10-15 April 2016.

[4] S. Geng, S. Liu, W. Hong, and X. Zhao, "Mm-wave $60 \mathrm{GHz}$ indoor channel parameters and correlation properties," Dianbo Kexue Xuebao/Chinese Journal of Radio Science, vol. 30, no. 4, pp. 808-813, 2015.

[5] N. Chahat, M. Zhadobov, R. Augustine, and R. Sauleau, "Human skin permittivity models for millimetre-wave range," Electronics Letters, vol. 47, no. 7, pp. 427-428, 2011.

[6] S. W. Harmer, N. Rezgui, N. Bowring, Z. Luklinska, and G. Ren, "Determination of the complex permittivity of textiles and leather in the $14-40 \mathrm{GHz}$ millimeter-wave band using a freewave transmittance only method," IET Microwaves, Antennas \& Propagation, vol. 2, no. 6, pp. 606-614, 2008.

[7] Z. Hongmei, Y. Hailong, and G. Shuting, "Research on path loss and shadow fading of ultra wideband simulation channel," International Journal of Distributed Sensor Networks, vol. 12, no. 12, pp. 1-7, 2016. 


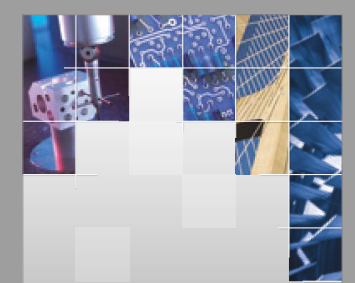

\section{Enfincering}
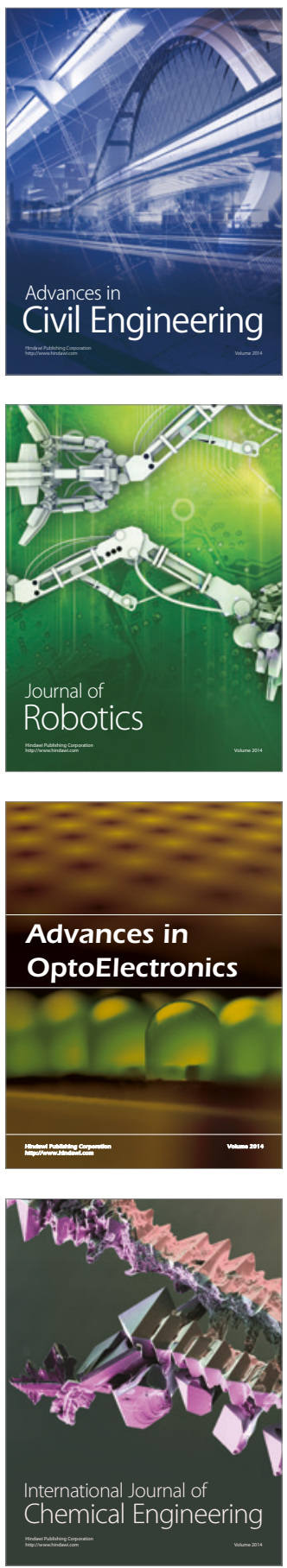

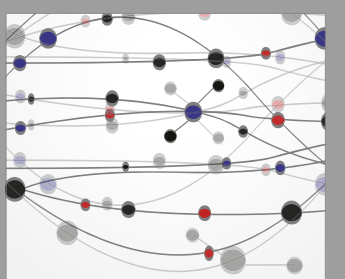

The Scientific World Journal

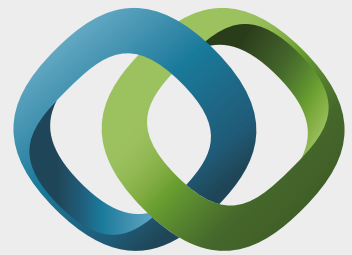

\section{Hindawi}

Submit your manuscripts at

https://www.hindawi.com
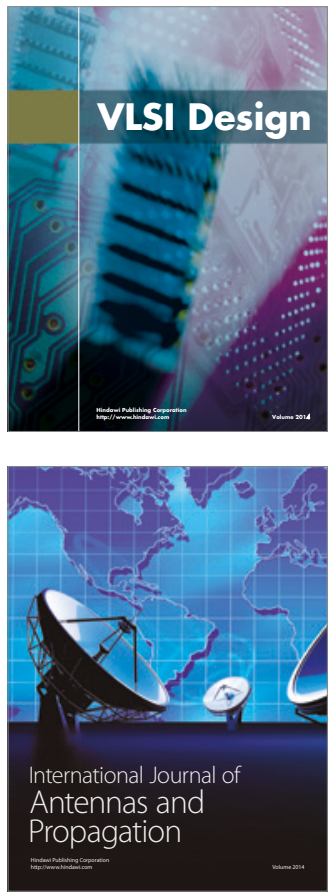

\section{Rotating}

Machinery
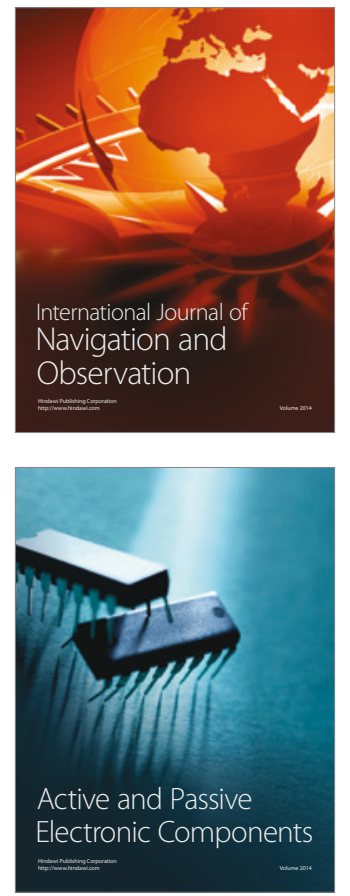
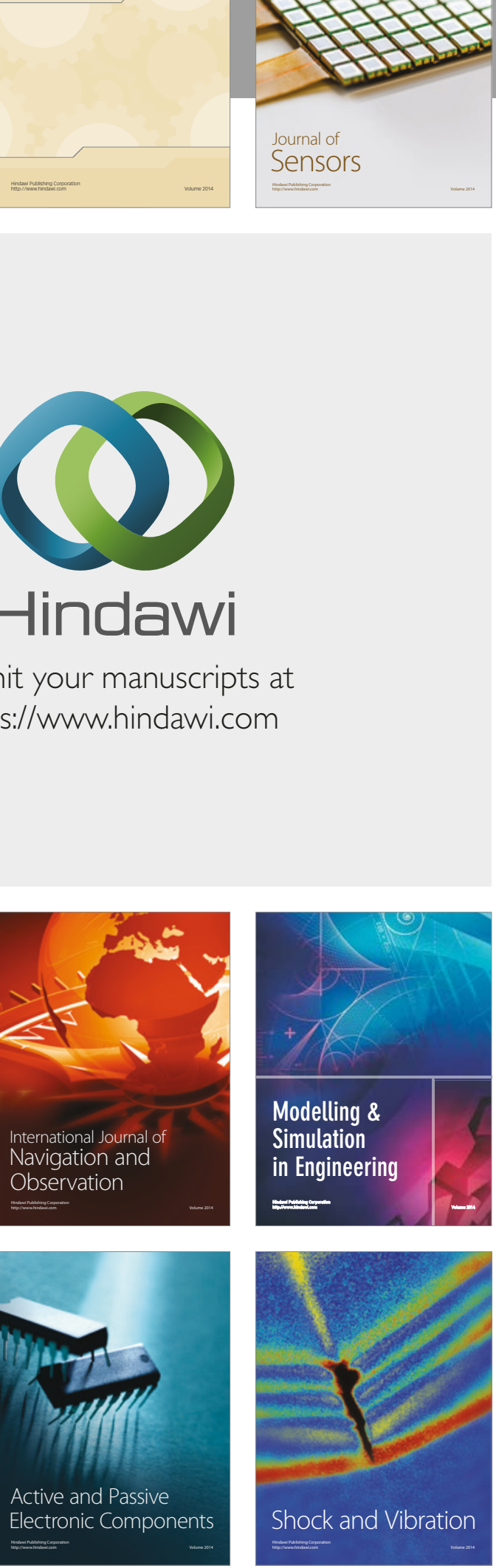
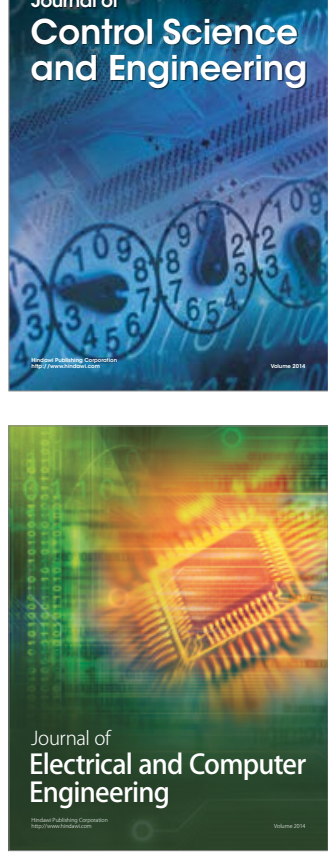

Distributed

Journal of

Control Science

and Engineering
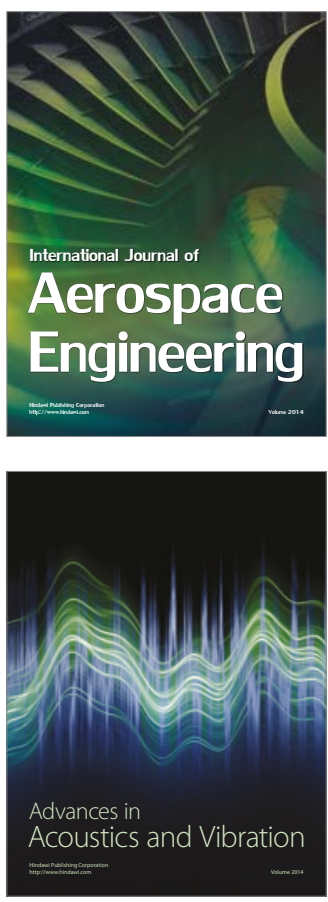

Sensor Networks 